2 National Institute for Health and Clinical Excellence. Dementia: Supporting People with Dementia and Their Carers in Health and Social Care (Clinical Guidelines, CG42). NICE, 2006.

Mustafa Alachkar, SpR in psychiatry, Manchester Mental Health and Social Care NHS Trust, Manchester, UK, email: mustafa.alachkar@mhsc.nhs.uk

doi: $10.1192 / \mathrm{pb} .38 .3 .137 \mathrm{a}$

\section{Exposure to acute child psychiatry presentations for core psychiatrists}

We are writing to draw attention to the lack of clarity provided by the Royal College of Psychiatrists regarding the role of the core trainee psychiatrist in assessing child and adolescent psychiatry patients out of hours. We believe it is important this issue is addressed as it confers broad implications for training, recruitment and service delivery. Crises of paediatric mental health tend to present out of hours. Ireland's 4th annual child and adolescent mental health service report details 'striking patterns in the number of [self-harm] presentations seen': 51\% of presentations were in the 8 -hour period of $7 \mathrm{pm}$ to $3 \mathrm{am}{ }^{1}$ This finding appears typical for paediatric psychiatry liaison services around the UK.

It is well known that in some trusts core trainees are excluded from child and adolescent mental health services (CAMHS)-led out-of-hours care pathways. This situation seems particularly unsatisfactory given that placements in developmental psychiatry are no longer obligatory. By failing to adequately furnish our future adult psychiatrists with skills in child and adolescent mental health, we are reinforcing a culture whereby young people are potentially falling through the care gap between CAMHS and adult mental health services. ${ }^{2,3}$ Indeed, this very issue is highlighted in a joint paper from the inter-faculty group of the child and adolescent psychiatry and the general and community psychiatry faculties which presents recommendations for the provision of psychiatric services to adolescents and young adults. ${ }^{4}$ Furthermore, by restricting the level of exposure to child psychiatry, we are doing little to encourage core trainees to perceive the specialty as a future career option.

As well as having an impact on the quality of training, the issue has far-reaching implications for patient care. The current lack of clarity fosters an atmosphere of uncertainty as situations arise where no one knows who holds responsibility to clerk a young person on arrival, thereby leading to potential delays in the patient being seen. Emergency department delays are a source of great concern to acute care trusts and create negative attitudes to psychiatric services in general. If we cannot manage to work in a safe and effective way, we are further contributing to the hostility not only towards our specialty but also to our patients, who are at their most vulnerable.

It is therefore our view that there should be an explicit expectation for core trainees to have exposure to the full range of acute psychiatric presentations, including child and adolescent patients, out of hours. It is of course essential that this experience would be supported by robust and accessible supervision structures in the form of a second on-call specialty trainee or consultant child psychiatrist. Although we recognise that the College is unable to tell trusts how to deliver their outof-hours services, it would be helpful if the core psychiatry curriculum contained more robust guidance as to the role of the core trainee in assessing child and adolescent psychiatry cases out of hours. Such a move would help to create clarity as well as holding local education providers to account.

\section{Declaration of interest}

R.C. sits on the College's Emergency Care Taskforce, which is currently considering the value of out-of-hours training.

1 Health Service Executive. Fourth Annual Child \& Adolescent Mental Health Service Report 2011-2012. HSE, 2012 (http://www.hse.ie/eng/services/ Publications/services/Mentalhealth/camhs20112012annualreport.pdf).

2 Singh SP. Transition of care from child to adult mental health services; the great divide. Curr Opin Psychiatry 2009; 22: 386-90.

3 Singh SP, Paul M, Ford T, Kramer T, Weaver T. Transitions of care from child and adolescent mental health services to adult mental health services (TRACK study): a study of protocols in Greater London. BMC Health Serv Res 2008; 8: 1-7.

4 Lamb C, Hill D, Kelvin R, Van Beinum M. Working at the CAMHS/Adult Interface: Good Practice Guidance for the Provision of Psychiatric Services to Adolescents/Young Adults. A Joint Paper from the Interfaculty Working Group of the Child and Adolescent Faculty and the General and Community Faculty of the Royal College of Psychiatrists, May 2008. Royal College of Psychiatrists, 2008.

Amanda K. Shine is ST6 in child and adolescent psychiatry and Rory Conn is ST4 in child and adolescent psychiatry, both at Tavistock and Portman NHS Foundation Trust, London, UK, email: amanda.shine@nhs.net, and Zaib Davids is consultant child and adolescent psychiatrist, Child and Adolescent Psychiatry Liaison Team, University College London Hospital. doi: $10.1192 / p b .38 .3 .138$

\section{Psychiatry for medical students: need for a more holistic approach to teaching?}

We are two medical students who wish to offer a perspective on undergraduate education and psychiatry.

During our student placement, we attended the old age psychiatry module at the Northern Deanery MRCPsych programme focusing on dementia and ethics. This was aimed at trainees and not specifically medical students but we were surprised to find that this was not above our level of knowledge. This prompted discussion of undergraduate psychiatry training more broadly, which we felt focused too heavily on the diagnosis of mental illness and less so on the holistic approach to the patient and their presentation as covered by the MRCPsych course. From our experience of undergraduate psychiatry we feel that the assessment by means of a logbook of conditions encourages students to find patients with a certain diagnosis, and in doing so overlooks the true essence of psychiatry. To our mind this incorporates the ability to consider all aspects of a patient's life and formulating these, while demonstrating compassion for another person at a time of most need.

Through choosing a 6-week placement in old age psychiatry we have been able to explore the specialty more thoroughly and broadly than facilitated within the standard undergraduate programme, and we have realised how little of psychiatry we have been exposed to as undergraduates. We have become more aware of the importance of considering the patient's personal and social circumstances alongside their diagnosis, and how these can influence each other. Specifically, the importance of a sound ethical approach to practice has been highlighted through the higher-level teaching we 